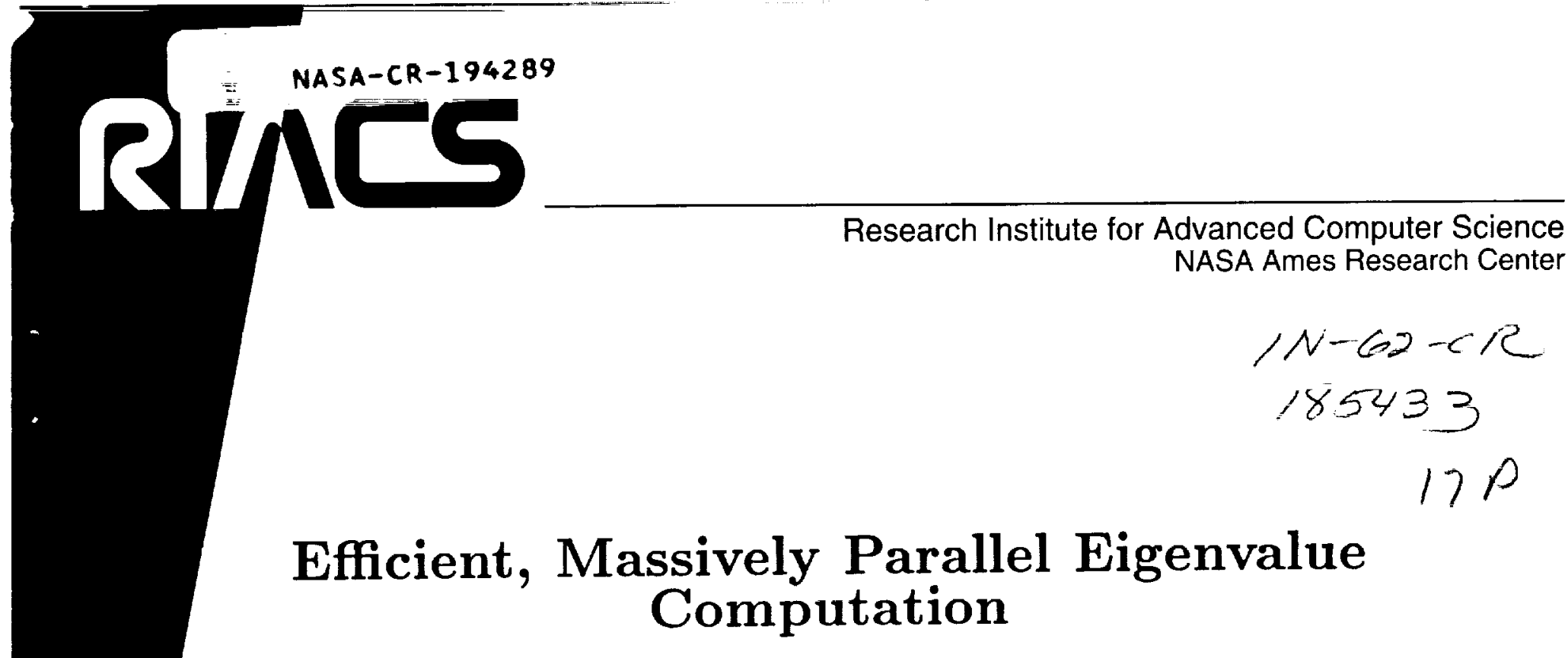

\author{
Yan Huo \\ Robert Schreiber
}

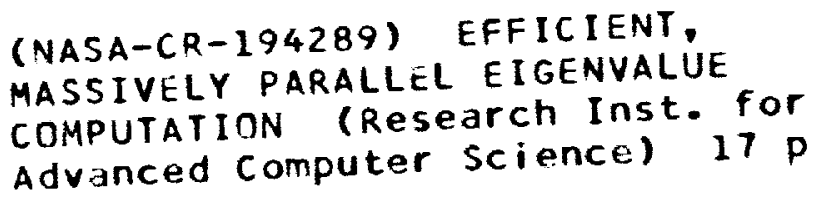

N94-13921

Unclas

G3/62 0185433

1

RIACS Technical Report 93.02 January, 1993

Submitted to: International Journal of Supercomputer Applications 



\title{
Efficient, Massively Parallel Eigenvalue Computation
}

\author{
Yan Huo \\ Robert Schreiber
}

The Research Institute of Advanced Computer Science is operated by Universities Space Research Association, The American City Building, Suite 311, Columbia, MD 21044, (301) 730-2656

Work reported herein was supported by NASA via Contract NAS 2-13721 between NASA and the Universities Space Research Association (USRA). Work was performed at the Research Institute for Advanced Computer Science (RIACS), NASA Ames Research Center, Moffett Field, CA 94035-1000. 



\title{
Efficient, Massively Parallel Eigenvalue Computation
}

\author{
Yan Huo* Robert Schreiber ${ }^{\dagger}$
}

January 22, 1993

\begin{abstract}
In numerical simulations of disordered electronic systems, one of the most common approaches is to diagonalize random Hamiltonian matrices and to study the eigenvalues and eigenfunctions of a single electron in the presence of a random potential. In this paper, we describe an effort to implement a matrix diagonalization routine for real symmetric dense matrices on massively parallel SIMD computers, the Maspar MP-1 and MP-2 systems. Results of numerical tests and timings are also presented.
\end{abstract}

"Department of Electrical Engineering, Princeton University, Princeton NJ 08544

${ }^{1}$ Research Institute for Advanced Computer Science, MS 230-5, NASA Ames Research Center, Moffett Field, CA 94035-1000. This author's work was supported by the NAS Systems Division and DARPA via Cooperative Agreement NCC 2-387 between NASA and the University Space Research Association (USRA). 


\section{Introduction}

Crystalline materials have been studied intensively by physicists since the beginning of quantum mechanics. Because of the periodicity of the crystalline structure, electronic wavefunctions can be classified as Bloch waves, and band structures can be calculated. However, disorder always exists in real materials, such as impurities, point defects, dislocations, etc. What has been gradually realized during the past thirty years is that disorder can play a very important role in determining the transport and thermodynamic properties of real materials. The concept of localization, introduced by Anderson in 1958 [1], states that if the disorder is very strong, the wave function may become localized, in that the envelop of the wavefunction decays exponentially from some point in space, i.e., $|\Psi(\mathbf{r})| \sim \exp \left(\left|\mathbf{r}-\mathbf{r}_{\mathbf{0}}\right| / \xi\right)$ and $\xi$ is the localization length.

One of the direct tests of the predictions of localization theory is to perform numerical solutions of the Schrodinger equation on a finite size system. By performing numerical diagonalization for random lattices of size $100 \times 100$, Yoshino and Okazaki [2] obtained a graphic demonstration of localized and extended states. Licciardello and Thouless [3] related the conductivity to the sensitivity of eigenvalues to changes in boundary conditions. Their numerical studies using this method provided one of the first hints that all states are localized in two dimensions with any amount of disorder.

The Quantum Hall Effect was probably one of the most important discoveries in condensed matter physics in the 80's. Von Klitzing, Dorda and Pepper [4] found that under certain conditions in a two-dimensional system of electrons subjected to a strong magnetic field, the Hall conductivity is quantized to integral multiples of $e^{2} / h$. It was soon recognized that localized states and extended states due to disorder play a key role in this phenomenon [5]. Since then, various numerical and analytic techniques have been applied to study this problem. Huo and Bhatt [6] have numerically diagonalized random systems at different sizes. By evaluating the topological Chern numbers of the electron wavefunctions and doing finite size scaling, they demonstrated that in the high magnetic-field limit, all states in the lowest Landau band are localized except at the single critical energy. They also obtained the critical exponent of the localization length close to this critical energy.

When dealing with disordered systems, analytic approaches are limited. Numerical simulations, especially numerical diagonalization, is expected to be one of the powerful alternatives to address these problems. However, the diagonalization of an $n \times n$ matrix required to describe a lattice of $n$ sites requires storage of order $n^{2}$ and number of floatingpoint operations (flops) of order $n^{3}$. Furthermore, since we are dealing with a random system, averaging over many impurity realizations is required. Thus the problem is extremely computation intensive. For instance, the calculation of Huo and Bhatt [6] was only made possible by an allocation of time on the Cray/2 from the National Center for Supercomputer Applications at the University of Mlinois. With recent advances in parallel computing technology, a natural question to ask is whether these simulations can be carried out efficiently on a massively parallel computer.

In this paper, we report on the implementation of a diagonalization routine for real symmetric dense matrices on a family of massively parallel, single-instruction, multiple- 
data (SIMD) computers, the Maspar MP-1 and MP-2. We find that a modification of the standard serial algorithm can be implemented efficiently on such a highly parallel SIMD machine. Moreover, despite the relatively low-level language, and a distributed memory, the software can be implemented easily because of a distributed-object-oriented programming style provided by the Maspar Linear Algebra Building Block (MPLABB) library.

This paper is organized as follows. Section II is an introduction to the MP-1 architecture. Section III describes the algorithm and its implementation. Section IV presents numerical results and timing data. The paper concludes with a discussion in Section V.

\section{The Maspar MP-x Systems}

The MP-1 and MP-2 architectures consist of the following two subsystems:

- The Array Control Unit (ACU)

- The Processing Element (PE) Array

The ACU is a 32-bit, custom integer RISC that stores the program, the instruction fetching and decoding logic, and is used for scalars, loop counters and the like. It includes its own private memory.

The PE array is a two-dimensional mesh of processors. Each processor may communicate directly with its 8 nearest neighbors. The processors at the edges of the mesh are connected by wrap-around channels to those at the opposite edge, making the array a two-dimensional torus. The two directions in the PE array are referred to as the $x$ and $y$ axes of the machine. The machine dimensions are referred to as nxproc and nyproc, and the number of processors is nproc $\equiv$ nyproc $\cdot$ nxproc. Thus, every PE has an $x$ coordinate ixproc and a $y$ coordinate iyproc, and also a serial number iproc $\equiv$ ixproc $+n x p r o c \cdot i y p r o c$.

Each PE in the array is a RISC processor with $64 \mathrm{~K}$ bytes of local memory. All PEs execute the same instruction, broadcast by the ACU. The machine clock rate is $12.5 \mathrm{MHz}$.

The set of all PE memories is called parallel memory or PMEM; it is the main data memory of the machine. Ordinarily, PMEM addresses are included in the instruction, so that all PEs address the same element of their local memory. By a memory layer we mean the set of locations at one PMEM address, but across the whole array of PEs. Each PE also includes 16 64-bit registers.

The hardware supports three communication primitives:

- ACU-PE communication;

- Nearest neighbor communications among the PEs;

- Communication in arbitrary patterns through a hardware global router.

These actions are expressed in the instruction set as synchronous, register-to-register operations. This allows interprocessor communication with essentially zero latency and very high bandwidth -200 Gbits per second for nearest neighbor communication, for example. 
On the MP-1, hardware arithmetic occurs on 4-bit data. Instructions that operate on $8,16,32$, and 64-bit integers and on 32 and 64-bit IEEE floating-point numbers are implemented in microcode. All operations occur on data in registers; only load and store instructions reference memory. Peak performance of a full $16 \mathrm{~K} \mathrm{MP-1}$ is 550 Mflops on IEEE 64-bit floating-point data. The MP-2 uses 32-bit hardware integer arithmetic, with microcode for higher precision and floating-point operations. Peak performance goes up to nearly 2000 Mflops in the MP-2.

Because floating-point arithmetic is implemented in microcode, it is slow relative to memory reference or interprocessor communication; these take less time than a multiplication. Thus, communication and memory traffic, traditional causes of low performance (relative to peak performance) on parallel machines, are not a serious obstacle here.

\section{Algorithm and Implementation}

The now standard serial algorithm [7] for computing the eigenvalues and eigenvectors of a real symmetric matrix consists of three phases:

1. Reduction of the real symmetric matrix $\mathbf{A}$ to tridiagonal form via orthogonal similarity transformations $\left(\mathbf{Q}^{T} \mathbf{A Q}=\mathbf{T}\right.$ where $\mathbf{Q}$ is orthogonal and $\mathbf{T}$ is real, symmetric, tridiagonal).

2. $Q R$ iteration to find all the eigenvalues and eigenvectors of the tridiagonal matrix $\mathbf{T}$, or bisection and inverse iteration if only few of the eigenvalues and(or) eigenvectors are needed $\left(\mathbf{V}^{T} \mathbf{T V}=\Lambda\right.$, where $\mathbf{V}$ has $k$ orthonormal columns and $\Lambda=\operatorname{diag}\left(\lambda_{1}, \ldots, \lambda_{k}\right)$ holds the desired eigenvalues).

3. Matrix multiplication to recover the eigenvectors of $A$ (the columns of $U=Q V$ ).

\subsection{Algorithms for the Parallel Computation of Eigensystems}

Parallel implementation of the $Q R$ iteration phase has proved to be quite difficult. For this reason, the Jacobi method for the symmetric eigenproblem has attracted much attention, because it is inherently parallel [8]. Unfortunately, the number of floating point operations required by the Jacobi method is much greater than the number required by methods based on tridiagonalization.

On many recent high-performance computers, both memory reference and interprocessor communication are much slower than arithmetic. This has led to the development and use of families of algorithms that exhibit locality of reference to data, as these algorithms make fewer memory and off-processor references per operation. For matrix computations, block algorithms, which break the computation down into tasks that operate on submatrices, have been very successful for these reasons. One advantage of the Jacobi method is that there are good block variants of this type $[9,10]$, while block tridiagonalization methods are less satisfactory. For these reasons, we first attempted an implementation of a variant of the 
standard algorithm. Since this implementation achieves high efficiency, we did not consider implementation of a Jacobi algorithm.

For the symmetric tridiagonal eigenvalue problem, both Cuppen's divide-and-conquer method [12] and bisection followed by inverse iteration have been advocated for the shared memory, multiprocessor environment [13]. While both methods provide both the eigenvalues and eigenvectors of the tridiagonal matrix, in both cases some subsequent orthogonalization step is needed for the eigenvectors corresponding to very close eigenvalues.

In an SIMD machine each processor executes exactly the same command at each cycle. Bisection and inverse iteration are well suited to the SIMD discipline, while the divide and conquer method appears to be less so. Also, at the time of our work, the accuracy of the divide and conquer method and means for its reliable implementation were still the subject of ongoing research [12]. We therefore chose bisection.

\subsection{MPL Programming on the Maspar}

Our code is written in the language MPL, a data-parallel variant of $\mathrm{C}$ provided by Maspar. In MPL, variables have a storage class, either singular or plural. Singular variables are traditional $C$ variables of any type. On the other hand, if $x$ is declared via plural double $x$, then there is an instance of $x$ on every PE, so that $x$ is implicitly an array. The operation $x *=2$; causes every PE to multiply its $x$ by 2 ; this is the only way to express parallelism in MPL.

The integer constants nxproc, nyproc, and nproc (which define the machine size) are predefined in MPL, as are the plural integer constants iyproc, ixproc, and iproc (which define processor coordinates).

Since matrix and vector data structures may involve more than nproc elements, several memory locations on each processor, organized as a plural array, are required for their storage. The MPL programmer must code the loops over these arrays required to implement simple matrix and vector operations such as the addition of two vectors. This process, akin to strip mining in vector machines with limited-length vector instructions (Cray machines, for example) is usually called virtualization; it is required to create a programming model in which there is one virtual processor per matrix or vector element. Since MPL (unlike Maspar Fortran, Fortran 90, or the $\mathrm{C}^{*}$ language developed by Thinking Machines Corporation) is not a virtual language, the MPL programmer ordinarily virtualizes by hand.

To simplify the coding of matrix and vector operations, Maspar provides a library (the MPLABB library) of simple operations on matrices and vectors of arbitrary size. The virtualization looping is hidden within this software layer. The types of data object supported are

- Vex. A one-dimensional array whose $i$ th element resides in processor ( $i$ mod nproc). In a vex $u$, the $x$ coordinate of the processor holding $u(i)$ varies most rapidly as $i$ increases. If the vector length is greater than nproc, multiple memory layers are used for its storage. If its length is $n$, then $n b \equiv(n+n p r o c-1) \div n$ proc layers are declared (plural double $\mathrm{u}[\mathrm{nb}]$ ); element $i$ is stored in memory layer $i \div$ nproc. 
- Vey. A one-dimensional array whose $i$ th element resides in the processor at coordinates $i$ yproc $=(i \bmod$ nyproc $)$ and $i x p r o c=(i \div$ nyproc $)$. In a vey $u$, the $y$ coordinate of the processor holding $u(i)$ varies most rapidly as $i$ increases.

- Mat. A two-dimensional array of arbitrary shape. In an $n y \times n x$ mat $A$, element $A(i, j)$ is stored on the processor at coordinates $i x p r o c=j \bmod n x p r o c$ and iyproc $=$ $i$ mod nyproc. The mapping to processors is referred to in the literature as the cyclic or torus wrap mapping, and in Maspar's jargon as the cut and stack mapping. (One may think of the matrix as being cut into nyproc $\times$ nxproc cookies, which are then stacked in parallel memory.) In this mapping, the matrix is viewed as a matrix of blocks. Each block is nyproc $\times$ nxproc. The number of blocks in the $y$ direction is $n b y \equiv\lceil(n y /$ nyproc $)\rceil$ and in the $x$ direction is $n b x \equiv\lceil(n x / n x p r o c)\rceil$. The blocks are then stored in memory as a plural array a in $x$-major order. Thus, the data declaration for such a mat would be plural double a [nby*nbx], and the scalar element $A(i, j)$ resides in the block at coordinates $y b l k \equiv i \div n y p r o c$ and $x b l k \equiv j \div n x p r o c$, which is contained in the plural array element $a[(y b l k * n b x)+x b l k]$.

- Blk. A sequence of nblks plural variables separated in memory by a fixed address increment. Thus

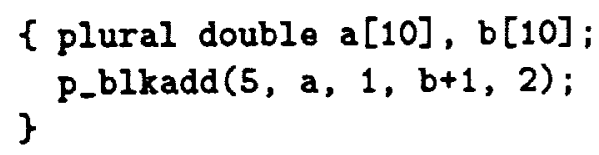

is the equivalent of

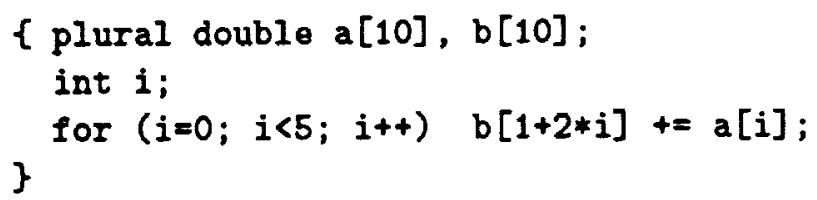

i.e. the data in all processors and at memory locations $a, a+1, a+2, a+3$, and $a+4$ are added to the data at memory locations $b+1, b+3, b+5, b+7$, and $b+9$.

Typical MPLABB routines used in the code are:

- p-veynrm2 Computes the Euclidean norm of a vector stored as a vey.

- p.mattovey Moves data from a column of a mat into a vey.

- pmatfromvey Broadcasts data from a vey into several consecutive columns of a mat.

- pmatsumtovey Sums the rows of a mat, placing the sums in a vey.

- p_blkaxpy Adds a scalar multiple of a sequence of memory layers to a second sequence of memory layers. 


\subsection{Implementation}

In this section we present a straightforward implementation of a parallel method based on tridiagonalization on the MP-x computers. Our codes can perform the following functions:

- Householder reduction of a real symmetric matrix to a tridiagonal matrix.

- Finding the eigenvalues and optionally the eigenvectors of a real symmetric tridiagonal matrix.

- Finding the eigenvalues and optionally the eigenvectors of a real symmetric matrix.

All eigenvalues, or those in an interval of the real axis, or all the eigenvalues from the $i$ th to the $j$ th largest can be computed.

The eigenvalues and eigenvectors of the tridiagonal matrix $\mathbf{T}$ are computed by an embarrassingly parallel algorithm employing bisection and inverse iteration methods. Bisection allows the computation of the $j$ th largest eigenvalue. Conceptually, processor $j$ uses bisection to find the $j$ th eigenvalue, in parallel with all the other processors. Having found its eigenvalue, processor $j$ then uses inverse iteration to find the corresponding eigenvector, if needed. Orthogonalization of the computed eigenvectors [15] is then performed as needed.

\subsection{Householder Tridiagonalization}

The Householder algorithm reduces an $n \times n$ matrix $\mathbf{A}$ to tridiagonal form by the application of $n-2$ orthogonal Householder transformations.

Algorithm 1: (Householder Tridiagonalization)

$$
\begin{aligned}
& \text { do } \begin{aligned}
k & =1 \text { to } n-2 \\
i & =n-k+1 \\
v & =\operatorname{house}(\mathbf{A}(1: i-1, i)) \\
p & =2 \mathrm{~A}(1: i-1,1: i-1) v / v^{T} v \\
w & =p-\left(p^{T} v\right) v / v^{T} v \\
& \text { /* Symmetric Rank-2 Update of } \mathbf{A} * / \\
\mathbf{A}(1: i-1,1: i-1)=\mathbf{A}(1: i-1,1: i-1)-v w^{T}-w v^{T} &
\end{aligned} \\
& \text { enddo }
\end{aligned}
$$

Here $v$ is the Householder vector at $k$ th stage:

$$
\begin{gathered}
v^{T}=\left(a_{i 1}, a_{i 2}, \ldots, a_{i, i-2}, a_{i, i-1}+\operatorname{sgn}\left(a_{i, i-1}\right) \sqrt{\sigma}\right) \\
\sigma=a_{1, i}^{2}+a_{2, i}^{2}+\ldots+a_{i-1, i}^{2} .
\end{gathered}
$$

Our implementation of tridiagonalization employs the natural parallelism of the process, which allows $O\left(n^{2}\right)$ operations to proceed in parallel. 
The Householder reduction starts in the $n^{\text {th }}$ column of $\mathbf{A}$, not the first. For a sequential machine, this does not make any difference. On the Maspar, however, this reduces the communication cost significantly. The reason is that at any stage $k, \mathrm{~A}$ is tridiagonal in its last $k-1$ rows and columns, and we only need to update the first $n-k$ rows and columns, which can be viewed as a submatrix with no offset.

The implementation is based on MPLABB routines. Matrices are stored as mats. Vectors as vexes and veys. At the $i$ th step, the elements $\mathbf{A}(1: i-1, i)$ are extracted and placed in a vey using p_mattovey. Computation of $\sigma$ uses the routine p_veynrm2. The vector $p$ is computed by a matrix-vector product routine that in turn uses p_blkaxpy and p.matsumtovey. Finally, the rank-2 update of $A$ uses p matfromvey, p matfromvex, and p_blkaxpy. Use of the MPLABB reduced the time required to develop our codes by a significant factor.

To reduce the communication overhead, array type conversions, e.g., conversion of a vector from vex form to vey form, have been minimized.

\subsection{Bisection}

The bisection procedure recursively halves an initial interval containing all eigenvalues (determined from the Gershgorin circle theorem). In our implementation of bisection, processor iproc computes eigenvalues indexed iproc, nproc+iproc, etc. A Sturm sequence is evaluated by a simple, stable, first-order recurrence to determine the number of eigenvalues less than or equal to the interval's midpoint. The half-interval containing the desired eigenvalue is then selected.

Each processor stores only the two boundary values of an interval containing the eigenvalue for which it is searching. To save memory, the diagonal and off-diagonal elements of the tridiagonal matrix are broadcast at each step.

If $n<$ nproc then load-balance is poor, since only processors $0, \ldots, n-1$ have anything to do. The cost of the bisection phase, however, is quite modest, so we don't view this as a real problem. An alternative scheme, which we have not implemented, would use $\mu \equiv\lfloor n$ proc $/ n\rfloor$ processors per eigenvalue when $n<$ nproc. The processors could subdivide the containing interval into $\mu+1$ equal subintervals, evaluate the Sturm sequence at the $\mu$ internal breakpoints simultaneously, and thereby contract the interval by a factor of $\mu$.

We have, in fact, now implemented a form of this idea. Initially, all the eigenvalues lie in one interval. We subdivide it into nproc +1 subintervals with nproc internal, equally spaced points $x_{i}, 1 \leq i \leq n$ proc. Processors $i$ then computes a Sturm sequence at $x_{i}$ to determine the number $\nu_{i}$ of eigenvalues to the left. Then, by communicating with processor $i-1$, processor $i$ computes $\nu_{i}-\nu_{i-1}$, which is the number of eigenvalues in the interval $\left(x_{i-1}, x_{i}\right]$. We then switch to the parallel bisection process above, with each processor now beginning with one of these smaller intervals. This first step, with one parallel evaluation of a Sturm sequence, reduces all the intervals by the factor nproc; it therefore saves $\log _{2} n p r o c$ subsequent steps. This reduced running time by roughly $20 \%$ on full $2^{14}$ processor machines. 


\section{Algorithm 2: (Parallel Bisection)}

For each processor iproc in PE array, the following commands are executed:

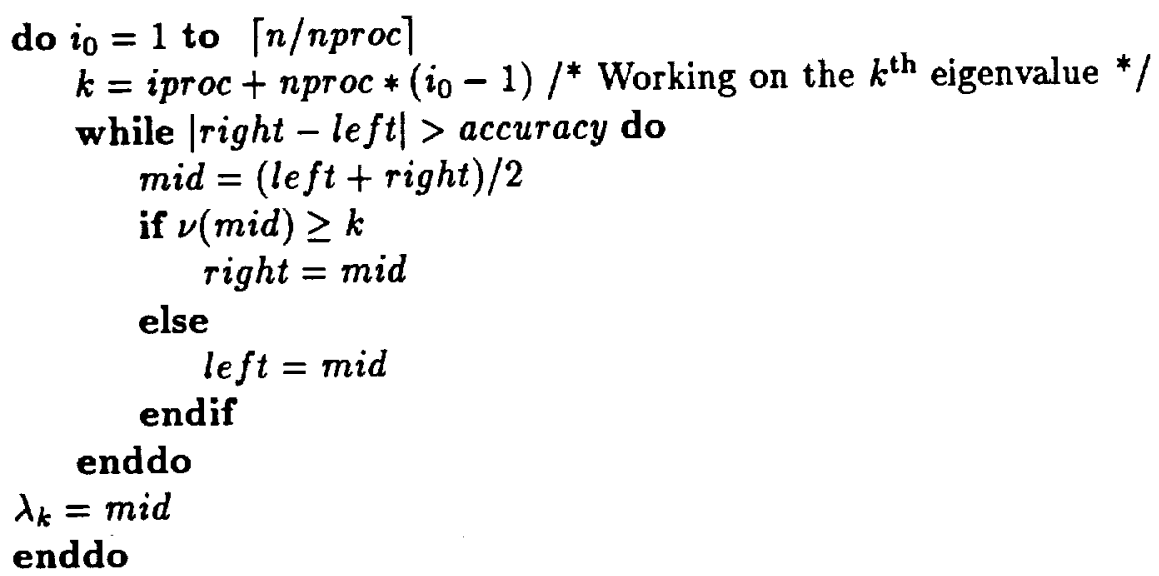

where $\nu(x)$ is the number of sign changes in the Sturm sequence evaluated at $x$, i.e. the number of eigenvalues less than or equal to $x$. In practice, the convergence test is not adaptive; rather we use $53-\log _{2}$ nproc iterations to achieve full IEEE double precision accuracy of 53 bits.

\subsection{Inverse Iteration}

Given an accurately computed eigenvalue $\lambda_{k}$, inverse iteration computes the corresponding eigenvector $\mathbf{u}_{k}$ by performing the power method with the shifted matrix $\left(\mathbf{T}-\lambda_{k} \mathbf{I}\right)^{-1}$. On a distributed memory parallel computer, the eigenvectors can be computed independently in each processor without any communication between the processors. Storage per processor may become a concern, as we discuss below.

\section{Algorithm 3: (Inverse Iteration)}

In processor $k \bmod n$ proc, the eigenvector $\mathbf{u}_{k}$ is computed as follows:

$$
\begin{aligned}
& \mathrm{x}_{0}=\mathrm{a} \text { random vector } \\
& \text { do } i=1 \text { to } m \\
& \quad \text { Solve }\left(\mathrm{T}-\lambda_{k} \mathrm{I}\right) \mathrm{x}_{i}=\mathrm{x}_{i-1} \\
& \text { enddo } \\
& \mathbf{u}_{k}=\mathrm{x}_{m} /\left\|\mathrm{x}_{m}\right\|_{2}
\end{aligned}
$$

where $\mathbf{T}$ is the tridiagonal matrix. We have found that $m \equiv 2$ iterations is sufficient to obtain full precision eigenvectors, even in the presence of closely spaced eigenvalues. The code solves the linear system using Gaussian elimination with partial pivoting, which causes one additional diagonal $\left(\mathrm{T}_{i, i+2}, 1 \leq i \leq n-2\right)$ to become nonzero. 
For the MP-x systems we have used, the number of processors ranges from $1 \mathrm{~K}$ to $16 \mathrm{~K}$; the local memory of each processor is fixed at 8192 words. This limited local memory constrained our initial implementation to matrices of order $n \leq 2048$, since several vectors of size $n$ are stored on every active processor.

The implementation uses $3 n$ words per processor: two superdiagonals of the upper triangular factor $\mathbf{U}$ in the $\mathbf{L} U$ decomposition of $\mathbf{T}-\lambda_{k} I$, and the vector $\mathbf{x}$. The factor $\mathbf{L}$ is not stored. To save memory, the elements of $T$ are broadcast to all the processors when needed.

The conventional implementation of Gaussian elimination would have used five arrays of length $n$. In the conventional $L U$ decomposition, the diagonal elements of $L$ are set to unity, so one vector is used to store it; three vectors store $U$, and one stores the right-hand side. In our implementation, we perform the forward substitution at the same time as the decomposition is carried out, so that the matrix $\mathrm{L}$ need not be saved. Moreover, we scale rows so that the diagonal of $U$ is unity. Then, only two diagonals of $U$ are stored per processor. This costs us some time, since we do two iterations with the same matrix, and we have to recompute the $L U$ decomposition the second time.

We found, however, that the resulting code still imposes too severe a limit on the size of the matrix that can be handled. Therefore, we recoded and "devirtualized" the code. In devirtualization, one makes several processors perform the work of one virtual processor in order to increase the available local memory. With this modification, we are able to handle the largest matrices that can fit in the machine.

Inverse iteration produces orthogonal eigenvectors for well-separated eigenvalues. With close eigenvalues, however, the corresponding eigenvectors have to be orthogonalized. As pointed out by Jessup and Ipsen [15], several factors might affect the accuracy of inverse iteration:

- The starting vector

- The reorthogonalization criterion

- The stopping criterion

Following their suggestion, we have used random vectors as starting vectors. The reorthogonalization criterion has been determined from our own numerical experiments: two adjacent eigenvalues $\lambda_{j}$ and $\lambda_{j+1}$ are considered close if $\left(\lambda_{j+1}-\lambda_{j}\right)<10^{-5}\|\mathrm{~T}\|_{R}$, where $\|\mathrm{T}\|_{R}=\max _{1 \leq j \leq n}\left(\left|\alpha_{j}\right|+\left|\beta_{j}\right|\right)$, where $\mathrm{T}$ has diagonal elements $\alpha_{1}, \ldots, \alpha_{n}$ and off-diagonal elements $\beta_{2}, \ldots, \beta_{n}$ and $\beta_{1}=0$. We have found that two iterations of inverse iteration followed by orthogonalization is sufficient for the problems of interest to us.

Our reorthogonalizer first identifies the clusters of close eigenvalues whose eigenvectors must be orthogonalized. These eigenvectors form groups of contiguous columns of the matrix of eigenvectors, because the bisection method produces sorted eigenvalues. We then use the Gram-Schmidt process to orthogonalize the vectors within these sets in parallel. The code again uses the MPLABB routines, storing the groups of vectors to be orthogonalized in mats. Groups are taken in decreasing order of size, and packed into a temporary mat, 
until the number of columns reaches nxproc. The code then performs the Gram-Schmidt process, working on several groups in parallel and on all columns within a group in parallel.

\section{Numerical Results and Timings}

We present experimental results on the accuracy and running time of our codes in this section.

To test the accuracy of the bisection and inverse iteration, we followed the suggestion of Jessup and Ipsen and used glued Wilkinson matrices [15] as a test case. A glued Wilkinson matrix $\mathbf{W}_{g}^{+}$is constructed as follows: The Wilkinson matrix $\mathbf{W}_{21}^{+}$of order $n=21$ has diagonal elements $10,9, \ldots, 1,0,1, \ldots, 9,10$ and immediate off-diagonal elements equal to one. It possesses pairs of eigenvalues that are very close [16]. The spacing between eigenvalues in a pair decreases with increasing magnitude of the eigenvalues, and the eigenvalues in the largest pairs are computationally coincident in double precision. A glued Wilkinson matrix $\mathbf{W}_{g}^{+}$is formed by placing copies of $\mathbf{W}_{21}^{+}$along the diagonal and setting off-diagonal elements equal to $10^{-14}$ at the positions $\beta_{21}, \beta_{42}, \ldots$ where the submatrices join. It is a difficult tridiagonal test cases for dealing with groups of close eigenvalues. A perturbed identity matrix $\mathbf{I}+\mathbf{E}$ is formed by adding to the identity matrix $\mathbf{I}$ a small random matrix $\mathbf{E}$, whose elements are random, uniformly distributed in $\left(-10^{-10}, 10^{-10}\right)$; its eigenvalues form a cluster close to one.

The accuracy of the computed eigendecomposition is determined by the residual $\mathcal{R}$ and deviation from orthogonality $\mathcal{O}$, which are defined as:

$$
\mathcal{R}=\left\|\mathbf{U}^{T} \mathbf{A} \mathbf{U}-\mathbf{\Lambda}\right\|_{F} / N, \quad \mathcal{O}=\left\|\mathbf{U}^{T} \mathbf{U}-I\right\|_{F} / N
$$

where $\|.\|_{F}$ denotes the Frobenius norm. Table 1 gives results for $\mathbf{W}_{g}^{+}$, Table 2 gives results for $\mathbf{I}+\mathbf{E}$.

In Table 3, we present timings results on an MP-1 system with $16 \mathrm{~K}$ processors for Householder tridiagonalization. All calculations were performed in IEEE 64-bit precision. We can see that as the matrix gets larger, performance improves; at the largest size studied, the code reached half the peak machine performance.

In Table 4, we present benchmark results for computation of all the eigenvalues and eigenvectors of a symmetric random matrix on the same system. Elements were taken independently from the uniform distribution on $(0,1)$, then symmetry imposed by adding the matrix to its transpose. The computation rate (in Mflops) is given assuming an operation count of $9 n^{3}$ flops; this is accurate for a $Q R$ method, but is an overestimate for our method.

In Figure 1, we compare our benchmark results on three Maspar systems (MP-1 with $16 \mathrm{~K}$ processors, MP-1 with $4 \mathrm{~K}$ processors, MP-2 with $1 \mathrm{~K}$ processors) with that of a single processor Cray Y/MP. The Cray results were produced using Cray-supported, optimized versions of the Eispack tridiagonalization routine TRED2 and the QR iteration code TQL2. We have also tested a modified Eispack code using bisection and inverse iteration to compute all the eigenvalues and eigenvectors which has the same operation count as ours. This code is slower than the QR code on the Cray, so we do not display these results. 


\section{Conclusion}

In this paper, we have described an implementation of matrix diagonalization on a massively parallel SIMD computer. We have demonstrated that massively parallel SIMD machines are very suitable for this kind of problem. Reliable, efficient, well-understood algorithms work well on these machines, which is a bit of a surprise. Also, despite the necessity of working with relatively low-level parallel programming tools, we were able to develop efficient codes readily through the use of a software layer for operation on distributed arrays. We expect that this approach will work well for many problems involving dense matrices and arrays.

\section{Acknowledgements}

Yan Huo acknowledges support from Electrical Engineering Department, Princeton University. Work at Princeton was performed under DEC DPRI grant and NSF CISE instrumentation grant CDA-9121709. He would also like to thank MasPar Computer Corp. for their hospitality during his visit. Robert Schreiber's work was supported by NASA under Contract NAS 2-13721 with the Universities Space Research Association. 


\section{References}

[1] Anderson, P. W. Phys. Rev. 109, 1492 (1958).

[2] Yoshino S. and Okazaki, M. J. Phys. Soc. Jpn. 43, 415 (1977).

[3] Licciardello, D. C. and Thouless, D. J. J. Phys. C 8, 4157 (1975).

[4] Von Klitzing, K., Dorda G., and M. Pepper, Phys. Rev. Lett. 45, 494 (1980). For a review, see Prange R. E. and Girvin, S. M. 1990, The Quantum Hall Effect. SpringerVerlag.

[5] Halperin, B. I., Phys. Rev. B 25, 2185 (1982).

[6] Huo, Y. and Bhatt, R. N. Phys. Rev. Lett. 68, 1375 (1992).

[7] Wilkinson, J. H., and Reinsch, C. 1971, Linear Algebra, vol. II of Handbook for Automatic Computation. New York: Springer-Verlag.

[8] Golub, G. H., and Van Loan, C. F., Matrix Computation. The Johns Hopkins University Press, second edition, 1989.

[9] Scott, D. S., Heath, M. T., and Ward, R. C., Lin. Alg. and Its Applic. 77, 345(1986).

[10] Shroff, G. and Schreiber, R., SIAM J. Matrix Anal. and Applics. 10 (1989).

[11] Maspar Mathematics Library (MPML) Reference Manual.

[12] Dongarra, J. J. and Sorensen D. C., SIAM J. Sci. Stat. Comput. 8, 139(1987).

[13] Ipsen, I. C. F., and Jessup, E. R., SIAM J. Sci. Stat. Comput. 11, 203(1990).

[14] see, for example, TINVIT in EISPACK.

[15] Jessup, E. R., and Ipsen, I. C. F., SIAM J. Sci. Stat. Comput. 13, 550(1992).

[16] Wilkinson, J. The Algebraic Eigenvalue Problem, Clarendon Press, Oxford, U.K., 1965. 
$\mathrm{N}$ : Order of matrix

$\mathcal{R}$ : Residual, $\left\|U^{T} A U-\Lambda\right\|_{F} / N$

$\mathcal{O}$ : Deviation from orthogonality, $\left\|U^{T} U-I\right\|_{F} / N$

Table 1: Numerical Results for Glued Wilkinson Matrix $\mathbf{W}_{g}^{+}$

\begin{tabular}{|lcc|}
\hline \hline $\mathrm{N}$ & $\mathcal{R}$ & $\mathcal{O}$ \\
\hline 105 & $8.1 \times 10^{-16}$ & $1.8 \times 10^{-16}$ \\
210 & $8.9 \times 10^{-16}$ & $1.9 \times 10^{-16}$ \\
315 & $1.5 \times 10^{-15}$ & $1.8 \times 10^{-16}$ \\
420 & $7.4 \times 10^{-16}$ & $1.2 \times 10^{-16}$ \\
525 & $1.6 \times 10^{-15}$ & $2.0 \times 10^{-16}$ \\
\hline
\end{tabular}

Table 2: Numerical Results for Perturbed Identity Matrix I + E

\begin{tabular}{|lcc|}
\hline \hline $\mathrm{N}$ & $\mathcal{R}$ & $\mathcal{O}$ \\
\hline 128 & $1.3 \times 10^{-16}$ & $1.2 \times 10^{-16}$ \\
256 & $1.3 \times 10^{-16}$ & $1.2 \times 10^{-16}$ \\
384 & $1.3 \times 10^{-16}$ & $1.1 \times 10^{-16}$ \\
512 & $1.3 \times 10^{-16}$ & $1.1 \times 10^{-16}$ \\
\hline
\end{tabular}

Table 3: Timing of Householder Tridiagonalization on $16 \mathrm{~K}$ Machine (Double Precision, Peak 550 MFLOPS)

\begin{tabular}{|lcc|}
\hline \hline $\mathrm{N}$ & TIME(sec) & MFLOP \\
\hline 512 & 6.3 & 57 \\
1024 & 23.2 & 123 \\
2048 & 118.2 & 194 \\
4096 & 746.6 & 245 \\
\hline \hline
\end{tabular}

Table 4: Timing of Diagonalization on $16 \mathrm{~K}$ Machine, (Double Precision, Peak $\sim 550$ MFLOPS)

\begin{tabular}{|lcc|}
\hline \hline$N$ & TIME & MFLOP \\
\hline 512 & 14.5 & 78 \\
1024 & 39.9 & 225 \\
2048 & 154 & 500 \\
4096 & 1477 & 418 \\
\hline
\end{tabular}




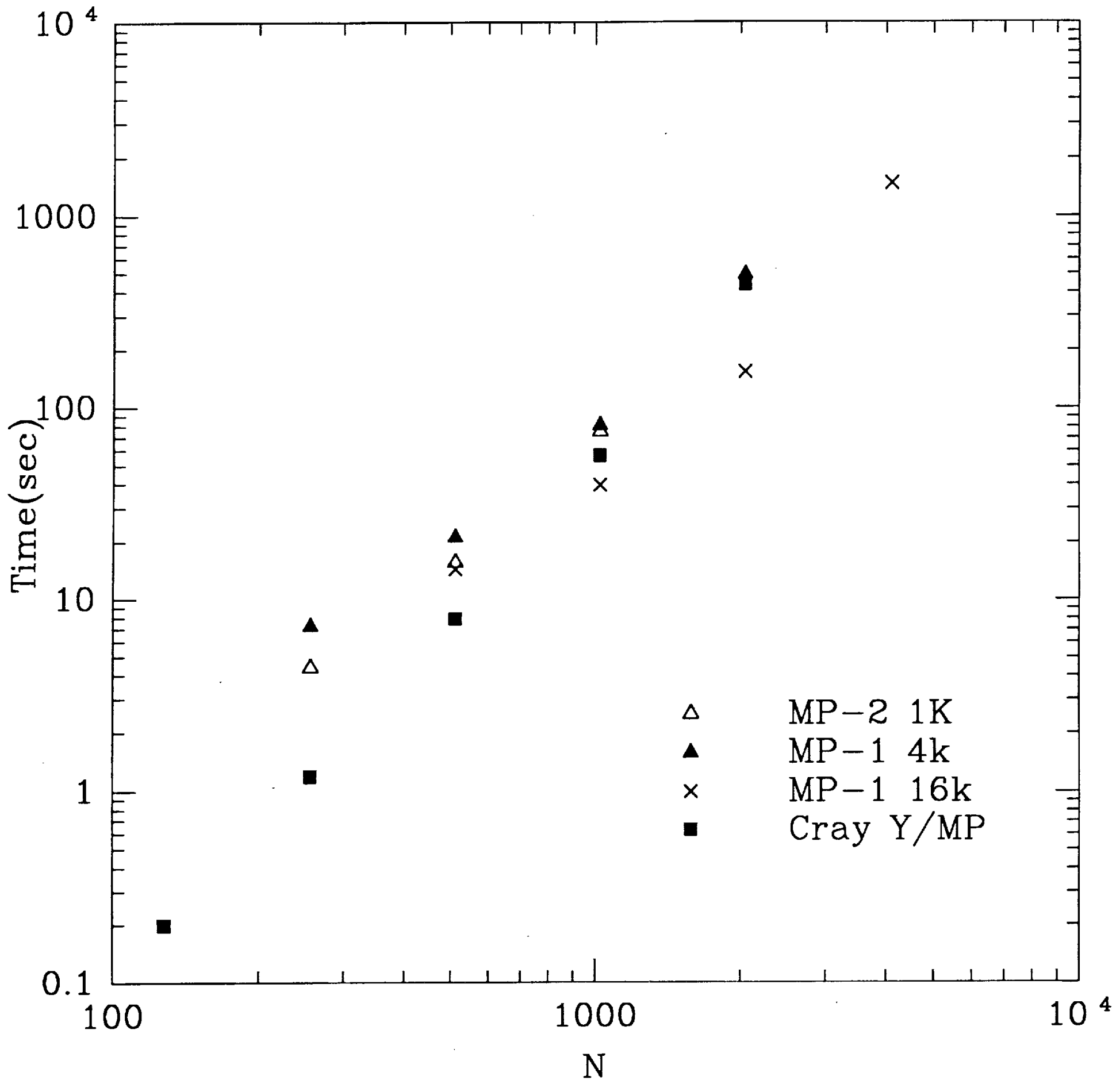


